\title{
Interpretação sísmica 3D com os atributos Semelhança e Envelope do reservatório de hidratos de metano do Blake Ridge (Carolina do sul, EUA)
}

Farias, K. C. C., Cruz, J.C.R., Universidade Federal do Pará

Copyright 2021, SBGf - Sociedade Brasileira de Geofísica

This paper was prepared for presentation during the $17^{\text {th }}$ International Congress of the Brazilian Geophysical Society held in Rio de Janeiro, Brazil, 16-19 August 2021.

Contents of this paper were reviewed by the Technical Committee of the $17^{\text {th }}$ International Congress of the Brazilian Geophysical Society and do not necessarily represent any position of the SBGf, its officers or members. Electronic reproduction or storage of any part of this paper for commercial purposes without the written consent of the Brazilian Geophysical Society is prohibited.

\section{Resumo}

Os atributos sísmicos Semelhança e Envelope foram usados para melhorar a identificação e distinção de feições sísmicas na região de hidratos de metano do Black Ridge na Carolina do Sul. As amostras do traço sísmico são consideradas como componentes de um vetor, e pelo cálculo da distância no hiperespaço define-se o atributo Semelhança, aprimorando-se definições de falhas. O atributo Envelope é calculado como o módulo da função complexa que representa o traço sísmico complexo. Usando a transformada de Hilbert, sendo proporcional linearmente com a impedância acústica. Conseguiu-se precisar características geológicas estudadas anteriormente e descobrir novas características. Apresentando um desempenho em maior parte satisfatório nas regiões de hidratos de metano.

\section{Introdução}

O dado sísmico foi obtido através de uma aquisição 3D e, após isto, foi submetido à fluxo de processamento para posterior interpretação, e está localizado no litoral da Carolina do Sul (EUA), na região de Black Ridge (Figura 1). O depósito de Black Ridge é o mais estudado do mundo principalmente devido a expressiva quantidade de hidratos de metano. O dado cedido por DGB Earth Sciences, foi adquirido usando a sísmica de reflexão, e analisou-se o padrão sísmico Bottom Simulate Reflectors (BSR), reflexão que se assemelha aproximadamente ao fundo do mar, causada pelo contraste entre hidratos de metano e sedimentos saturados de gás subjacentes por meio do contraste de alta impedância. Além da sísmica de reflexão, que é um dos métodos indiretos mais usados para a caracterização de reservatórios de hidratos de metano, ainda existem o ecobatímetro, perfilagem geofísica de poços e métodos geoelétricos (Aguiar, 2017). O Reservatório de hidratos de metano de Black Ridge é caracterizado como expansivo, porém diluído, com hidratos de metano sem advecção significativa de fluido. $\mathrm{E}$ é conhecido como uma das maiores províncias de hidratos gasoso de margem passiva na Terra. A quantidade de hidratos de metano retido na província ainda é especulativa (Hornbach et al., 2008), e representa a capacidade de se tornar uma nova fonte de energia com magnitude de potencial energético significativa (Gandara et al., 2015). Além disso, os hidratos de metano podem estar ligados a eventos de aquecimento global, sua liberação pode resultar em concentrações elevadas de carbono atmosférico, desencadeando ou aumentando o aquecimento global (Hornbach et al., 2008). Após testar alguns atributos sísmicos utilizados na caracterização de reservatórios, escolheu-se dois deles que tiveram melhor desempenho para a interpretação do dado, Semelhança e Envelope. O atributo Semelhança é uma forma de "coerência" que expressa o quanto dois ou mais segmentos são parecidos. Uma Semelhança de 1 significa que os segmentos de rastreio são completamente idênticos em forma de onda e amplitude. Uma Semelhança de 0 significa que eles são completamente diferentes (Böniger e Tronicke, 2010). O atributo Envelope é matematicamente expresso pelo valor absoluto do sinal analítico. $O$ uso de ambos atributos foi eficiente para a interpretação da feição BSR e identificação de anomalias possivelmente ligadas à presença de hidratos de metano.

\section{Metodologia}

No atributo Semelhança, a similaridade é dada matematicamente como 1 menos a distância euclidiana entre os vetores dividida pela soma do comprimento de cada vetor. O comprimento de um vetor é sua norma, também chamada de valor RMS:

$$
\operatorname{sim}=1-\frac{\sqrt{\sum_{i=1}^{N}(X i-Y i)^{2}}}{\sqrt{\sum_{i=1}^{N} X i^{2}}+\sqrt{\sum_{i=1}^{N} Y i^{2}}}
$$

Em que $X$ e $Y$ são dois vetores e $\mathrm{N}$ o comprimento destes

$$
0<\operatorname{sim}<1
$$

Como o atributo Semelhança aprimora regiões com falhas, pode-se visualizar as regiões que aprisionam hidratos de metano, ou vazios, que podem indicar o escape dos hidratos.

O atributo sísmico Envelope é descrito por:

$$
A(t)=\sqrt{s(t)^{2}+h(t)^{2}}
$$

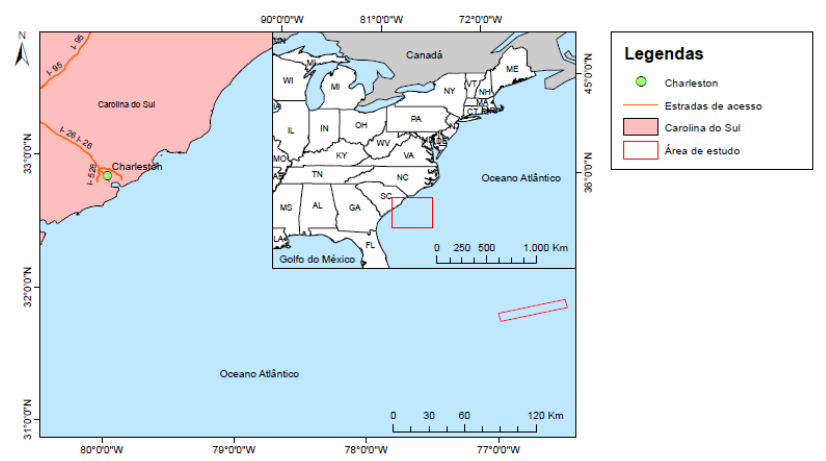

Figura 1. Localização do dado sísmico 3D (Black Ridge, South Carolina) 
Em que $s(\mathrm{t})$ é o sinal registrado/parte real e $\mathrm{h}(\mathrm{t})$ é a transformada de Hilbert de $\mathrm{s}(\mathrm{t})$. O Envelope, por ser independente da fase e sensível a alterações em impedância acústica, enfatiza mudanças de amplitude da seção sísmica gerada que estão ligadas a acumulação do gás em alguns casos.

\section{Resultados}

O dado sísmico (Figura 2) foi limitado a um intervalo de $3400 \mathrm{~ms}$ à aproximadamente $5000 \mathrm{~ms}$, região que se obteve as feições estudadas. Do ponto de vista sedimentar, os resultados da perfuração revelam siltitos e argilitos de grão fino quase uniformes, 700 metros abaixo do fundo do mar, e os resultados de sondagem sísmica indicam que essas condições sedimentares existem em toda área de estudo (Paul et al., 1996).

\section{Análise do atributo Envelope}

Sobre as feições encontradas (Figura 3), traçou-se um limite de fase de hidrato de metano em lilás para o primeiro trecho, e roxo para o segundo trecho. Em verde escuro, traçou-se a onda de sedimentos de flanco oriental (OSFO), e as lentes em verde claro.

O BSR traçado faz parte de estudos anteriores que mostraram sua extensão por $76 \%$ do volume do dado, e aproximadamente $50 \%$ da área da província de hidratos. $\mathrm{O}$ atributo Envelope mostrou no primeiro trecho, poucas áreas com reflexões sísmicas contínuas de alta amplitude associadas ao hidratos e ao gás livre em relação ao trecho dois e as lentes. O segundo trecho, mostrou-se com extensão bem maior do que estudos anteriores sugerem, e com 0 atributo Envelope teve comportamento expressivo, com zonas elevadas do in-line 1 ao 11, e zonas rebaixadas do in-line 12 ao 14, o que gerou um efeito de fendas na Figura 3.B mas que pode ser visto com clareza por um outro ângulo na Figura 5. Esse rebaixamento do BSR no trecho dois pode estar ligado a processos de erosão e sedimentação com taxas suficientemente altas a ponto de impactar a temperatura subsuperficial, levando ao desequilíbrio do BSR. E, ainda pode ser um indicativo de que a alteração no gradiente térmico faz com que o sistema de hidrato de gás seja dinâmico como proposto por Hornbach et al. (2008).

Sabendo que a ocorrência do gás requer condições propícias de temperatura e pressão, isso poderia levar à liberação de metano, que explicaria a ausência de gás na região onde se formaram fendas (Figura 3.B), e presença de gás abaixo do BSR que sofreu deslocamento. Além disso, analisando o comportamento da BSR além do intervalo de estudo, percebe-se que a descontinuidade do trecho um para o trecho dois tende a diminuir no volume, aparecendo no intervalo de 1 a 14 como dois trechos e a partir do in-line 58 como um trecho apenas. Mostrando novamente uma possível movimentação das linhas do BSR.

A OSFO acontece em todas as 96 linhas do volume, e mostra uma brusca interrupção do segundo trecho do BSR. Uma explicação proposta por Hornbach et al. (2008) é que os sedimentos com gás apresentam maior porosidade/permeabilidade do que os estratos circundantes, sugerindo uma advecção ascendente do BSR e o fluxo contínuo de fluído ao longo do limite de ondas de sedimentos.

$\mathrm{Na}$ região em que se traçou as lentes (refletor que cruza estratos), notou-se a presença de anomalias de alta amplitude que indicam a presença de sedimentos contendo quantidades significativas de hidratos. As lentes se estendem de norte ao sul, com uma área de $30 \mathrm{~km}^{2}$. Pode-se notar que a morfologia das lentes se dispõe como uma trapa que concentra de forma eficaz os hidratos de metano.

Além das lentes, encontrou-se um veio estreito (Anton et al. 1999) no conjunto de dados 3D ao serem mapeados. Entretanto, com o Envelope aplicado ao dado, houve uma imprecisão para traçar os pontos do veio no intervalo de

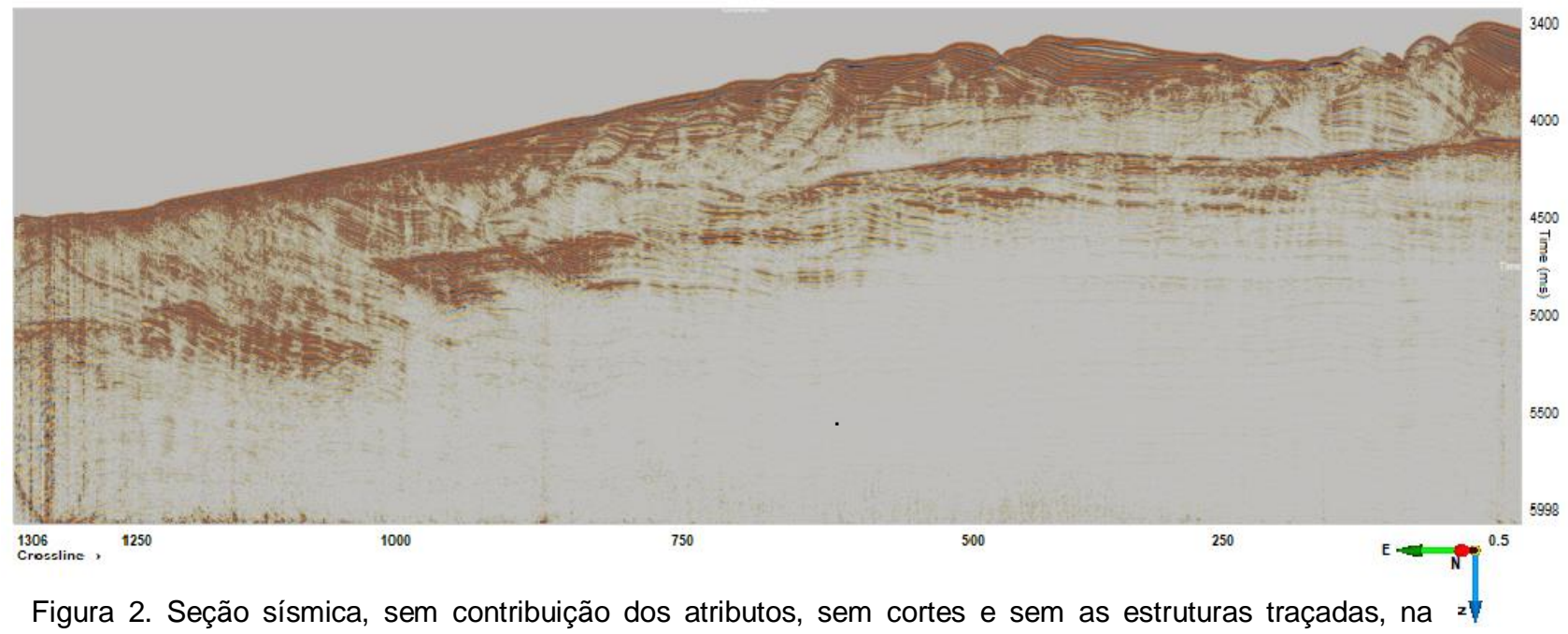
localização do in-line 11. 

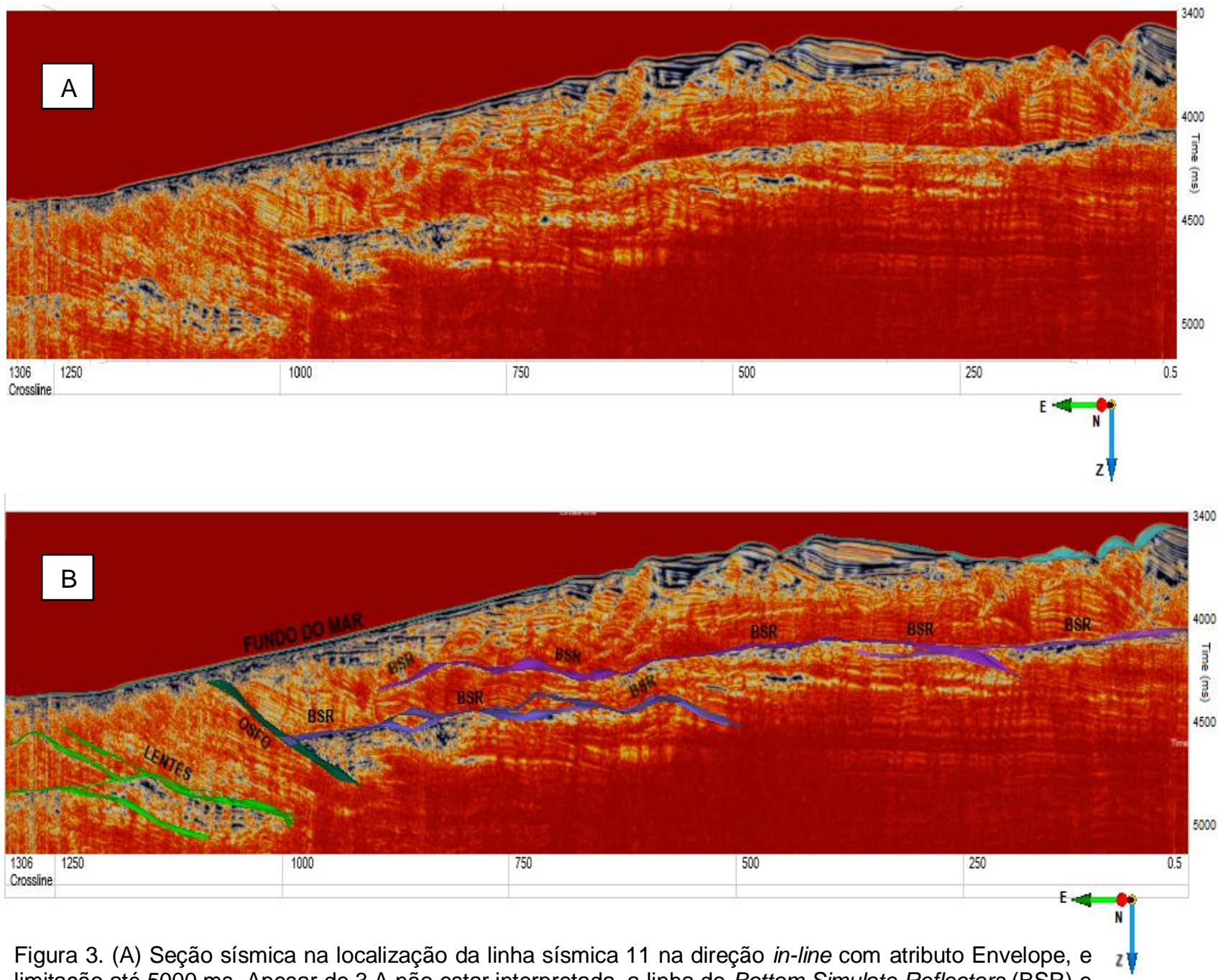

limitação até $5000 \mathrm{~ms}$. Apesar de 3.A não estar interpretada, a linha do Bottom Simulate Reflectors (BSR) e da Onda de Sedimentos de Flanco Oriental (OSFO) é de fácil identificação. (B) Seção sísmica interpretada com as estruturas encontradas destacadas. Em azul o fundo do mar, em roxo e lilás a feição BSR, em verde uma OSFO, e em verde claro as lentes. A Barra de cores escolhida para a visualização do atributo Envelope foi a Seismic. As colorações vermelhas, alaranjadas, amarelas e pretas variam respectivamente da menor para a maior anomalia, pois, o atributo Envelope realça as altas amplitudes que estão relacionadas aos hidratos de gás.

estudo. Em algumas linhas o veio parecia bem marcado, e em outras aparecia de forma sutil e se confundia com o primeiro trecho do BSR. Isso pode ter acontecido devido ao veio intersectar o BSR ao longo de sua borda oeste (Hornbach et al. 2008) e ser muito mais marcante para visualização no intervalo a partir do in-line 58 , fora do intervalo aqui estudado. Ainda assim, mesmo sem a visualização clara do veio, ele está presente e de acordo com a análise de inversão de forma de onda de anomalias de amplitude similares (característica dos veios), há a possibilidade de concentração de até $42 \%$ de hidratos (Gorman et al., 2002; Korenaga et al., 1997).

Além do que já foi dito, o Envelope ainda pôde realçar as regiões "blindadas", entre $4300 \mathrm{~ms}$ e $5000 \mathrm{~ms}$, a parte mais clara ao oeste da seção (Figura 3.B), característica sísmica que indica presença de hidratos de gás. Os hidratos de gás em suas zonas de estabilidade muitas vezes causam a cimentação dos sedimentos aonde se situam, essa cimentação reduz os contrastes de impedância acústica entre as camadas com texturas diferentes. A cimentação juntamente com os hidratos de gás imprime assim um aspecto "blindado" (Barros, 2009).

\section{Análise do atributo Semelhança}

Para a análise do atributo Semelhança no dado, optou-se por sua aplicação à direção crossline que contém 1306 linhas ao todo, e observou-se seu comportamento nas linhas que se localizam próximas às feições traçadas neste trabalho. Nas regiões em que a linha do crossline cruzava as feições, o atributo Semelhança mostra alta concentração de características indicativas de gases. 

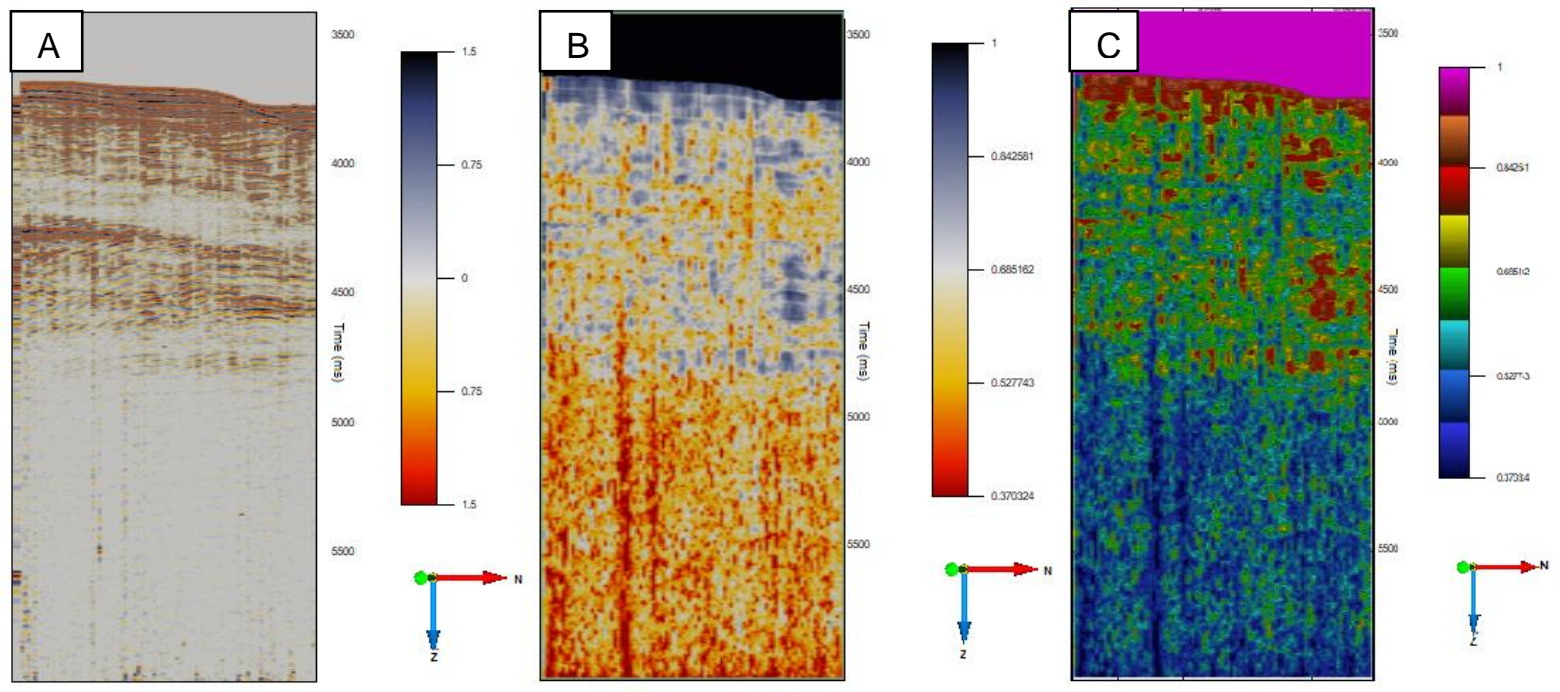

Figura 4. Esquema de seções na direção Crossline na linha 654. A) Seção sísmica. B) Seção com o atributo Semelhança com barra de cores Seismics. C) Seção com atributo Semelhança com barra de cores Contour Gradients. As seções estão todas sem cortes, para mostrar o contraste das cores mesmo fora das regiões de interesse. A região de A) abaixo de aproximadamente $5000 \mathrm{~ms}$ mostra-se com raros registros de reflexões, que são melhor visualizados progressivamente nas Figuras B e C. Nas regiões em que havia limite de fase de hidratos de metano, no intervalo de $4300-4800 \mathrm{~ms}$, o atributo mostra uma cor de verde à vermelho, indicando uma Semelhança muito próxima a 1 (segmento de rastreio com mesma forma de onda e amplitude). Onde a Semelhança era muito próxima ou igual a 0 (forma de onda e amplitude diferente) a visualização é mais próxima da cor azul.

Essa concentração aparecia em grande volume principalmente nas lentes e no primeiro trecho do BSR. As porções mais superficiais da seção mostram características similares às das regiões de hidratos de metano devido predominância de lâmina d'água, que tem mesma forma de onda e amplitude, aparecendo na seção como similaridade próxima ou igual à 1. Observa-se também, que ao utilizar a seção sem cortes abaixo de $5000 \mathrm{~ms}$, obteve-se com o atributo Semelhança distinções com valores maiores que a metade de uma similaridade.

Isso mostra que 0 atributo Semelhança, tem comportamento superior ao do atributo Envelope, podendo ser visto com clareza na Figura 4.C, também realçando as regiões blindadas causadas possivelmente pela cimentação advindas de hidratos de metano, e marca descontinuidades presente no dado. Faz então, separações claras das similaridades na seção, que são realçadas com a barra de cores Contour Gradients, que mostra variações em visualização e número mais exatas que na Figura 4.B. A Figura 5 ainda enfatiza a similaridade que ocorre abaixo das feições BSR e sua disposição ao longo das linhas na direção Crossline, por meio da visualização dos padrões em amarelo, vermelho e marrom dentro do intervalo de 4300 a 4800 ms.

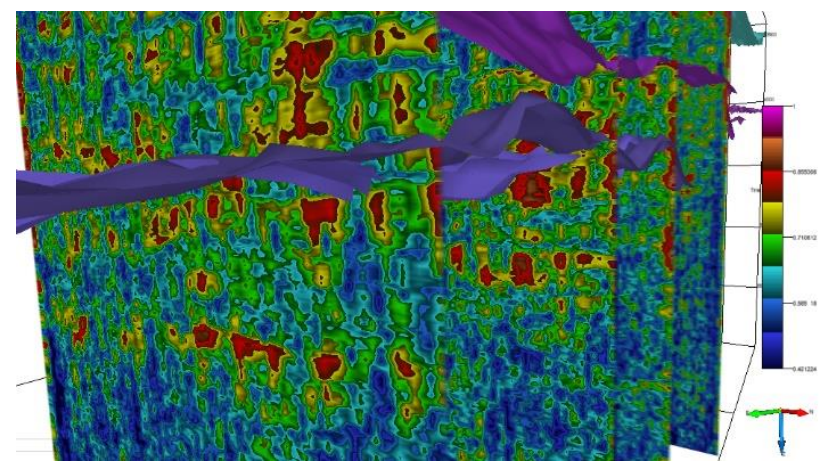

Figura 5. Esquema de seções com o atributo Semelhança na direção Crossline, com linhas de posição 354, 454, 554 e 654. Com feições BSR no trecho 1 e 2 cruzando a seção. 


\section{Discussão e Conclusões}

$\mathrm{O}$ atributo Envelope mostrou-se um bom discriminante de características geológicas, e de acumulações de gás. Pode-se perceber que provavelmente o sistema de hidratos de metano de Black Ridge é dinâmico como proposto por Hornbach et al. (2008) devido ao rebaixamento traçado no trecho dois do BSR após a aplicação do atributo Envelope, e pelo deslocamento ao longo do volume estudado que indica que em alguma região da província o BSR pode ser apenas um trecho contínuo. $\mathrm{E}$ ainda, mostrou que o trecho dois é maior do que se conseguia visualizar.

A análise do atributo Semelhança no deslocamento da linha na direção crossline atrelado ao Envelope na linha 11 da direção in-line mostrou a possível disposição da concentração dos hidratos. Um estudo mais detalhado, usando 0 atributo Semelhança, pode ajudar na investigação da ocorrência de escape de hidratos de gás, pois, com esse atributo é possível identificar a existência de condutos de fluido, que se ligados às concentrações podem gerar uma fuga em larga escala, acarretando em possíveis problemas ambientais.

\section{Agradecimentos}

Para DGB Earth Sciences pelos dados cedidos e pelo software OpendTect 6.0 Free. Para Marcos Flávio Costa Medeiros graduando de Geologia da Universidade Federal do Pará pelas discussões e pela confecção do mapa de localização. E para todos que contribuíram para 0 desenvolvimento deste trabalho.

\section{Referências}

Aguiar, L. F., 2017. Análise de atributos na identificação de feições sísmicas associadas à presença de hidratos de gás na bacia da Foz Do Amazonas. Trabalho de conclusão de curso da Universidade Federal Fluminense. Niterói, Brasil: $10-24$.

Barros, C. S., 2009. Análise de Atributos Sísmicos Para Caracterização de Hidratos de Gás no Cone do Rio Grande. Trabalho de conclusão de curso da Universidade Federal Fluminense. Niterói, Brasil: $26-41$.

Böniger, U.,Tronicke, J., 2010. Improving the interpretability of 3D GPR data using target-specific attributes: application to tomb detection. Journal of Archaeological Science. Vol. 37.

Brown, A. R., 1999. Interpretation of Three-Dimensional Seismic Data. The American Association of Petroleum Geologists and the Society of Exploration Geophysicists. Tulsa, Oklahoma, U.S.A. 5 $5^{\text {a }}$ ed. $147-186$.

dGB Earth Sciences B.V, 2016. Introduction To OpendTect \& OpendTect Pro. Manual de Treinamento. 78 - 123.

Holbrook et al. (6 co-autores), 2002. Escape of methane gas through sediment waves in a large methane hydrate province. vol. 30 ; no. $5 ; 467-470$.
Hornbach et al. (4 co-autores), 2008. Three-dimensional seismic imaging of the Blake Ridge methane hydrate province: Evidence for large, concentrated zones of gas hydrate and morphologically driven advection. Journal Of Geophysical Research, Vol. 113: 1 - 13.

Michelena J. R., et al. (2 co-autores), 1998. Similarity analysis: A new tool to sumarize seismic attributes information. PDVSA-Intevep. The Leading Edge, Society of Exploration Geophysicist. Vol. 17.

Taner, M. T., F. Koehler, and R. E. Sheriff, 1979. Complex seismic trace analysis: Geophysics, Vol. 44: 1041 - 1063. 